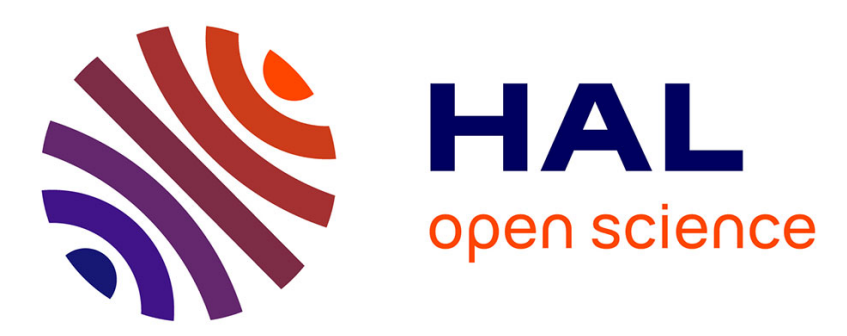

\title{
Optimal Control Problems with Partially Polyhedric Constraints
}

\author{
J. Frederic Bonnans, Hasnaa Zidani
}

\section{To cite this version:}

J. Frederic Bonnans, Hasnaa Zidani. Optimal Control Problems with Partially Polyhedric Constraints. [Research Report] RR-3349, INRIA. 1998. inria-00073340

\section{HAL Id: inria-00073340 https://hal.inria.fr/inria-00073340}

Submitted on 24 May 2006

HAL is a multi-disciplinary open access archive for the deposit and dissemination of scientific research documents, whether they are published or not. The documents may come from teaching and research institutions in France or abroad, or from public or private research centers.
L'archive ouverte pluridisciplinaire HAL, est destinée au dépôt et à la diffusion de documents scientifiques de niveau recherche, publiés ou non, émanant des établissements d'enseignement et de recherche français ou étrangers, des laboratoires publics ou privés. 
INSTITUT NATIONAL DE RECHERCHE EN INFORMATIQUE ET EN AUTOMATIQUE

\section{Optimal control problems with partially polyhedric constraints}

J. Frédéric Bonnans, Housnaa Zidani

$\mathbf{N}^{\circ} 3349$

Janvier 1998

THÈME 4 



\title{
Optimal control problems with partially polyhedric constraints
}

\author{
J. Frédéric Bonnans* ${ }^{*}$ Housnaa Zidani ${ }^{\dagger}$ \\ Thème 4 - Simulation et optimisation \\ de systèmes complexes \\ Projet PROMATH \\ Rapport de recherche $\mathrm{n}^{\circ} 3349$ - Janvier 1998 - 20 pages
}

\begin{abstract}
This paper discusses a class of state constrained optimal control problems for which it is possible to formulate second order necessary or sufficient conditions that do not involve all curvature terms for the constraints. This kind of result is classical in the case of polyhedric control constraints. Our theory of optimization problems with partially polyhedric constraints allows to extend these results to the case when the control constraints are polyhedric, and there are also some state constraints. The analysis is based on the assumption that some strict semilinearized qualification condition is satisfied. We apply the theory to some optimal control problems of elliptic equations with state and control constraints.
\end{abstract}

Key-words: Optimal control, elliptic systems, sensitivity analysis, expansion of solutions, second order optimality conditions, Legendre forms, polyhedricity.

(Résumé : tsvp)

\footnotetext{
* Projet PROMATH, INRIA. Email: Frederic.Bonnans@inria.fr.

$\dagger$ UFR Math, Lab MIP, Université Paul Sabatier, 118 Route de Narbonne, 31062 Toulouse. Email: zidani@mip.ups-tlse.fr
} 


\section{Problèmes de commande optimale sous contraintes partiellement polyédriques}

Résumé : Cet article discute une classe de problèmes de commande optimale pour lesquels il est possible d'énoncer des conditions nécessaires du second ordre qui ne font apparaître qu'une partie des termes de courbure des contraintes. Ce type de résultat est classique dans le cas de contraintes polyédriques sur la commande. Notre théorie des problèmes partiellement polyédriques étend ces résultats au cas où les contraintes polyédriques sur la commande sont combinées avec des contraintes sur l'état. L'analyse est basée sur une hypothèse dite de condition de qualification stricte semilinéarisée. Nous appliquons ces résultats à des problèmes de commande optimale d'équations elliptiques avec des contraintes sur la commande et sur l'état.

Mots-clé : Commande optimale, systèmes elliptiques, analyse de sensibilité, développement des solutions, conditions d'optimalité du deuxième ordre, formes de Legendre, polyédricité. 
AMS subject classifications Primary 49K40, Secondary 49K20, 35B30, 35J60, 90C31.

\section{Introduction}

This study is motivated by optimal control problems that have local control constraints and a finite number of state constraints. The problem was considered recently in [15], where second order necessary optimality conditions were obtained. The aim of this paper is to show that this result is a particular case of a general property of abstract optimization problems that have two types of constraints, the first of them being polyhedric. Polyhedricity theory for convex sets is a classical tool for obtaining formulas for the directional derivative of the projection over a convex set [18, 25], was applied to nonlinear control problems [30, 23], and has been linked to the recent work on sensitivity analysis for abstract optimization problems $[6,8,10]$.

The paper is organized as follows. Section 2 presents a theory of second order necessary or sufficient optimality conditions for abstract optimization problems that satisfy the strict semilinearized qualification condition. In the case corresponding to an optimal control problem with polyhedric control constraints and a finite number of additional inequality constraints, the theory is complete in the sense that there is no gap between the necessary and sufficient conditions. More precisely, we obtain a characterization of the quadratic growth condition.

In section 3, assuming a weak second order sufficient condition, and the strict semilinearized qualification condition, we provide a formula for computing the directional derivative of the optimal control (as well as a second order expansion of the value function) with respect to a perturbation.

The last section discusses the application of the previous results to some optimal control problems of elliptic equations. We consider the case of nonnegative control subject to a finite number of state constraints.

Notations Let $(P)$ be an optimization problem. By $F(P), \varepsilon-S(P)$ and val $(P)$, we denote the feasible set, set of $\varepsilon$ solutions and value of problem $(P)$, respectively.

\section{Second order abstract optimality conditions}

In this section we discuss the theory of second order optimality conditions for optimization problems of the following type:

$$
\operatorname{Min}_{x} f(x) ; x \in K_{X} ; G(x) \in K_{Y} .
$$

Here $X$ and $Y$ are Banach spaces, $K_{X}$ and $K_{Y}$ are closed convex subsets of $X$ and $Y$, respectively, and $f$ and $G$ are twice continuously differentiable mappings from $X$ into $\mathbb{R}$ and $Y$. We remind that, if $\mathcal{K}$ is a convex subset of a Banach space $\mathcal{X}$, and $x \in \mathcal{K}$, then the tangent and normal cones, $T_{\mathcal{K}}$ and $N_{\mathcal{K}}$, and the cone of feasible directions $\mathcal{R}_{\mathcal{K}}$, are defined 
as

$$
\begin{aligned}
T_{\mathcal{K}}(x) & :=\{y \in \mathcal{X} ; \exists x(\sigma)=x+\sigma y+o(\sigma) \in \mathcal{K}, \sigma \geq 0\} \\
N_{\mathcal{K}}(x) & :=\left\{x^{*} \in \mathcal{X}^{*} ;\left\langle x^{*}, y\right\rangle \leq 0, \forall y \in T_{\mathcal{K}}(x)\right\}, \\
\mathcal{R}_{\mathcal{K}}(x) & :=\{y \in \mathcal{X} ; \exists t>0 ; x+t y \in \mathcal{K}\}
\end{aligned}
$$

with the convention that these sets are empty if $x \notin \mathcal{K}$. An interesting case is when $K_{X}$ is polyhedric in the following sense $[25,18]$.

Definition 1 Let $x_{0} \in K_{X}$ and $x^{*} \in N_{K_{X}}\left(x_{0}\right)$. We say that $K_{X}$ is polyhedric at $x_{0}$ for the normal direction $x^{*}$ if

$$
T_{K_{X}}\left(x_{0}\right) \cap\left(x^{*}\right)^{\perp}=\overline{\mathcal{R}_{K_{X}}\left(x_{0}\right) \cap\left(x^{*}\right)^{\perp}} .
$$

If $K_{X}$ is polyhedric at each $x_{0} \in K_{X}$ for all $x^{*} \in N_{K_{X}}\left(x_{0}\right)$, we say that $K_{X}$ is polyhedric.

By setting

$$
\mathcal{K}:=K_{X} \times K_{Y}, \quad \mathcal{Y}:=X \times Y, \quad \mathcal{G}(x):=(x, G(x)),
$$

we can write the abstract optimization problem $(A P)$ under the form

$$
\operatorname{Min}_{x} f(x) \text { s.t. } \mathcal{G}(x) \in \mathcal{K},
$$

with $\mathcal{G}(x)$, twice continuously differentiable mapping from $X$ into $\mathcal{Y}$, and $\mathcal{K}$ closed convex subset of $\mathcal{Y}$. We will use several times the relationship between the two formats, in order to use the results that were derived for problem $(A P 2)$. For instance, the standard constraint qualification condition for $x_{0} \in F(A P 2)$, due to Robinson [26], is as follows:

$$
0 \in \operatorname{int}\left\{D \mathcal{G}\left(x_{0}\right) X-\left(\mathcal{K}-\mathcal{G}\left(x_{0}\right)\right)\right\} \text {. }
$$

Lemma 2.1 (Robinson [26]). Let $x_{0} \in F(A P 2)$ satisfy (2.2). Then the following metric regularity property holds. There exists $\varepsilon>0$ and $\alpha>0$ such that, for all $x \in B\left(x_{0}, \varepsilon\right)$ there exists $\hat{x} \in \mathcal{G}^{-1}(\mathcal{K})$ satisfying

$$
\|\hat{x}-x\| \leq \alpha \operatorname{dist}(\mathcal{G}(x), \mathcal{K}) .
$$

It is easy to show (e.g. [10]) that the qualification condition for a problem of the form $(A P)$ (after it has been put under the form $(A P 2)$ ) is equivalent to

$$
0 \in \operatorname{int}\left\{D G\left(x_{0}\right)\left(K_{X}-x_{0}\right)-\left(K_{Y}-G\left(x_{0}\right)\right)\right\} .
$$

The critical cone at $x_{0} \in F(A P)$ is defined as the set of directions of decrease of the cost function that are tangent to the feasible set. More precisely,

$$
C\left(x_{0}\right):=\left\{h \in T_{K_{X}}\left(x_{0}\right) ; D f\left(x_{0}\right) h \leq 0 ; D G\left(x_{0}\right) h \in T_{K_{Y}}\left[G\left(x_{0}\right)\right]\right\} .
$$

The Lagrangian function and the set of Lagrange multipliers are defined as

$$
\begin{aligned}
\mathcal{L}(x, \lambda) & :=f(x)+\langle\lambda, G(x)\rangle, \\
\Lambda(x) & :=\left\{(q, \lambda) \in N_{K_{X}}(x) \times N_{K_{Y}}[G(x)] ; D_{x} \mathcal{L}(x, \lambda)+q=0\right\} .
\end{aligned}
$$


Lemma 2.2 (Zowe-Kurcyusz [31]). Let $x_{0}$ be a local solution of $(A P)$ satisfying the qualification hypothesis (2.2). Then with $x_{0}$ is associated a non empty and bounded set of Lagrange multipliers.

It is convenient to use the following well-known characterization of the critical cone.

Lemma 2.3 Let $\Lambda(x) \neq \emptyset$, say contains $(q, \lambda)$. Then $D f(x) h=0$ whenever $h \in C(x)$, and

$$
C(x)=\left\{h \in T_{K_{X}}(x) \cap q^{\perp} ; D G(x) h \in T_{K_{Y}}[G(x)] \cap \lambda^{\perp}\right\} .
$$

Proof.Let $h \in X$ be tangent to the feasible set of $(A P)$, in the sense that $h \in T_{K_{X}}(x)$ and $D G(x) h \in T_{K_{Y}}[G(x)]$. By definition of $\Lambda(x)$, we have

$$
0=\left\langle D_{x} \mathcal{L}(x, \lambda)+q, h\right\rangle=D f(x) h+\langle\lambda, D G(x) h\rangle+\langle q, h\rangle .
$$

Since the last two terms are nonpositive, we have $D f(x) h \geq 0$, and $D f(x) h \leq 0$ iff the last two terms are zero. The result follows.

Let $x \in F(A P)$. Using the above lemma, we may view the critical cone as a linearization of the following set

$$
A(q, \lambda):=\left\{h \in\left(K_{X}-x\right) \cap q^{\perp} ; D G(x) h \in T_{K_{Y}}[G(x)] \cap \lambda^{\perp}\right\} .
$$

Note that in this expression we chose to "linearize" the constraint $G(x) \in K_{Y}$, but not the relation $x \in K_{X}$. The set $A(q, \lambda)$ is the inverse image, through the linear continuous mapping $h \rightarrow(h, D G(x) h)$, of the closed convex set

$$
\left(\left(K_{X}-x\right) \cap q^{\perp}\right) \times\left(T_{K_{Y}}[G(x)] \cap \lambda^{\perp}\right) .
$$

We will use the associated qualification condition, that we will call the strict semilinearized qualification condition (we justify this terminology below). From the above discussion, it follows that the expression of the strict semilinearized qualification condition is

$$
0 \in \operatorname{int}\left\{D G(x)\left(\left(K_{X}-x\right) \cap q^{\perp}\right)-T_{K_{Y}}[G(x)] \cap \lambda^{\perp}\right\} .
$$

We may compare this condition to the more classical strict qualification condition, introduced in [28] (see also [4]), whose expression, for problem $(A P)$, is

$$
0 \in \operatorname{int}\left\{D G(x)\left(\left(K_{X}-x\right) \cap q^{\perp}\right)-\left(K_{Y}-G(x)\right) \cap \lambda^{\perp}\right\} .
$$

Lemma 2.4 (i) Condition (2.6) implies (CQA), and both conditions are equivalent if $K$ is a polyhedron.

(ii) Assume that the standard constraint qualification (2.2) holds. Then condition $(C Q A)$ implies existence and uniqueness of the Lagrange multiplier. 
Proof.(i) Since $\left(K_{Y}-G(x)\right) \subset T_{K_{Y}}(G(x))$, we obviously have that (2.6) implies $(C Q A)$. Assume now that $K$ is finite dimensional, and that $(C Q A)$ holds. Since $\mathbb{R}_{+}\left(K_{Y}-G(x)\right)=$ $\mathcal{R}_{K_{Y}}(x)$ is equal to $T_{K_{Y}}(G(x))$, conditions $(C Q A)$ and (2.6) are obviously equivalent.

(ii) It is known that the strict qualification condition (2.6) implies existence and uniqueness of the Lagrange multiplier, see [28]. Since $(C Q A)$ is nothing but the strict qualification condition after linearization of the second constraint (that leaves invariant the set of Lagrange multipliers), we obtain that the set of Lagrange multipliers, that by $(2.2)$ is non empty, is in fact a singleton.

It is possible to express a second order necessary optimality condition for problem $(A P)$, using the result of [16], in term of the second order tangent set to $K \subset Y$, at $y \in K_{Y}$ in direction $z \in T_{K_{Y}}(y)$, that is defined as

$$
T_{K_{Y}}^{2}(y, z):=\left\{w \in Y ; y+t z+\frac{t^{2}}{2} w+o\left(t^{2}\right) \in K_{Y}, t \geq 0\right\} .
$$

Let $x_{0}$ be a qualified local minimum of $(A P)$. Set

$$
\mathcal{T}(h):=T_{K_{Y}}^{2}\left[G\left(x_{0}\right), D G\left(x_{0}\right) h\right] .
$$

Theorem 2.1 Assume that $K_{X}$ is polyhedric. Let $x_{0}$ be a qualified local minimum of $(A P)$ satisfying the strict semilinearized qualification condition $(C Q A)$. Then

(i) $C\left(x_{0}\right) \cap \mathcal{R}_{K_{X}}\left(x_{0}\right)$ is a dense subset of $C\left(x_{0}\right)$, and each $h \in C\left(x_{0}\right) \cap \mathcal{R}_{K_{X}}\left(x_{0}\right)$ satisfies

$$
D_{x^{2}}^{2} \mathcal{L}\left(x_{0}, \lambda\right)(h, h)-\sigma(\lambda, \mathcal{T}(h)) \geq 0 .
$$

(ii) If in addition $h \rightarrow \sigma(\lambda, \mathcal{T}(h))$ is lower semi continuous over $C\left(x_{0}\right)$, then (2.7) holds for all critical direction $h$.

(iii) If $K_{Y}$ is a polyhedron, then for all critical direction $h$ we have

$$
D_{x^{2}}^{2} \mathcal{L}\left(x_{0}, \lambda\right)(h, h) \geq 0 .
$$

Proof.(i) Step a. We claim that $C\left(x_{0}\right) \cap \mathcal{R}_{K_{X}}\left(x_{0}\right)$ is a dense subset of $C\left(x_{0}\right)$. Let $h \in C\left(x_{0}\right)$, and fix $\varepsilon>0$. Since $K_{X}$ is polyhedric, there exists $\hat{h}_{\varepsilon} \in \mathcal{R}_{K_{X}}\left(x_{0}\right) \cap q^{\perp}$ such that $\left\|\hat{h}_{\varepsilon}-h\right\| \leq \varepsilon$. Let $t_{\varepsilon}>0$ be such that $x_{0}+t_{\varepsilon} \hat{h}_{\varepsilon} \in K_{X}$. We use the metric regularity property that, by lemma 2.1, follows from $(C Q A)$ :

There exists $\gamma>0$ and $\alpha>0$ such that, if $\hat{w} \in X$, and $\|\hat{w}\| \leq \gamma$, then there exists $w \in\left(K_{X}-x_{0}\right) \cap q^{\perp}$ such that

$$
\begin{aligned}
& D G\left(x_{0}\right) w \in T_{K_{Y}}\left[G\left(x_{0}\right)\right] \cap \lambda^{\perp}, \text { and } \\
& \|w-\hat{w}\| \leq \alpha\left[\operatorname{dist}\left(\hat{w},\left(K_{X}-x_{0}\right) \cap q^{\perp}\right)+\operatorname{dist}\left(D G\left(x_{0}\right) \hat{w}, T_{K_{Y}}\left[G\left(x_{0}\right)\right] \cap \lambda^{\perp}\right)\right] .
\end{aligned}
$$

Reducing $t_{\varepsilon}$ if necessary, we have that $\hat{w}_{\varepsilon}:=t_{\varepsilon} \hat{h}_{\varepsilon}$ is such that $\left\|\hat{w}_{\varepsilon}\right\| \leq \gamma$ and $\hat{w}_{\varepsilon} \in\left(K_{X}-\right.$ $\left.x_{0}\right) \cap q^{\perp}$. Since

$$
\operatorname{dist}\left(D G\left(x_{0}\right) \hat{w}_{\varepsilon}, T_{K_{Y}}\left[G\left(x_{0}\right)\right] \cap \lambda^{\perp}\right)=O\left(\varepsilon t_{\varepsilon}\right),
$$


it follows that there exists $w_{\varepsilon} \in\left(K_{X}-x_{0}\right) \cap q^{\perp}$ such that

$$
\begin{aligned}
& D G(x) w_{\varepsilon} \in T_{K_{Y}}\left[G\left(x_{0}\right)\right] \cap \lambda^{\perp}, \\
& \left\|w_{\varepsilon}-\hat{w}_{\varepsilon}\right\|=\alpha \operatorname{dist}\left(D G\left(x_{0}\right) \hat{w}_{\varepsilon}, T_{K_{Y}}\left[G\left(x_{0}\right)\right] \cap \lambda^{\perp}\right)=O\left(t_{\varepsilon} \varepsilon\right) .
\end{aligned}
$$

Set $h_{\varepsilon}:=t_{\varepsilon}^{-1} w_{\varepsilon}$. Then $h_{\varepsilon} \in C\left(x_{0}\right) \cap \mathcal{R}_{K_{X}}\left(x_{0}\right)$, and $\left\|h_{\varepsilon}-h\right\|=O(\varepsilon)$. This proves our claim. Step b. Since $x_{0}$ is a qualified local solution of $(A P)$, and the Lagrange multiplier is unique, by [16, Thm 4.1], the following second order necessary condition holds: for any critical direction $h$, we have

$$
D_{x^{2}}^{2} \mathcal{L}\left(x_{0}, \lambda\right)(h, h)-\sigma\left(q, T_{K_{X}}^{2}\left(x_{0}, h\right)\right)-\sigma(\lambda, \mathcal{T}(h)) \geq 0 .
$$

On the other hand, since $q \in N_{K_{X}}\left(x_{0}\right)$, we have $\sigma\left(q, T_{K_{X}}^{2}\left(x_{0}, h\right)\right) \leq 0$ [16, Section 4]. If $h \in \mathcal{R}_{K_{X}}\left(x_{0}\right)$, then $0 \in T_{K_{X}}^{2}\left(x_{0}, h\right)$, so that $\sigma\left(q, T_{K_{X}}^{2}\left(x_{0}, h\right)\right)=0$. Point (i) follows.

(ii) Let $h \in C\left(x_{0}\right)$. By (i) there exists $h_{k} \rightarrow h, h_{k} \in C\left(x_{0}\right) \cap \mathcal{R}_{K_{X}}\left(x_{0}\right)$, and

$$
D_{x^{2}}^{2} \mathcal{L}\left(x_{0}, \lambda\right)\left(h_{k}, h_{k}\right) \geq \sigma\left(\lambda, \mathcal{T}\left(h_{k}\right)\right) .
$$

Since the right hand side is l.s.c. by hypothesis, and $D_{x^{2}}^{2} \mathcal{L}\left(x_{0}, \lambda\right)(\cdot, \cdot)$ is a continuous function, we may pass to the limit in this inequality. Point (ii) follows.

(iii) If $K_{Y}$ is a polyhedron, then it is well known that $0 \in \mathcal{T}(h)$ (e.g. [10]), whence $\sigma(\lambda, \mathcal{T}(h))=0$, for all critical direction $h$. The result follows then from (ii).

In order to formulate second order sufficient conditions, we need the following concept.

Definition 2 (See e.g. [19]). We say that a quadratic form $Q$ on a Hilbert space $X$ is a Legendre form if $Q$ is weakly l.s.c. and, whenever a sequence $\left\{x_{k}\right\} \subset X$ satisfies $x_{k} \stackrel{w}{\rightarrow} x$ and $Q\left(x_{k}\right) \rightarrow Q(x)$, then $x_{k} \rightarrow x$.

The function $x \rightarrow\|x\|^{2}$ is the simplest example of a Legendre form. More generally, if $N>0$ and $Q$ is a weakly continuous quadratic form, it is easy to check that $x \rightarrow$ $N\|x\|^{2}+Q(x)$ is a Legendre form, see e.g. [11].

Definition 3 We say that $x_{0}$ is a local solution of $(A P)$ satisfying the quadratic growth condition if

$$
\exists \alpha>0 ; \quad F(x) \geq F\left(x_{0}\right)+\alpha\left\|x-x_{0}\right\|^{2}+o\left(\left\|x-x_{0}\right\|^{2}\right), \quad \forall x \in F(A P) .
$$

Theorem 2.2 Assume that $K_{X}$ is a polyhedric subset of the Hilbert space $X$. Let $x_{0}$ be a qualified local minimum of $(A P)$ satisfying the strict semilinearized qualification condition $(C Q A)$, and let $\left(q_{0}, \lambda_{0}\right)$ be the unique associated Lagrange multiplier. If $Q_{0}(h):=$ $D_{x^{2}}^{2} \mathcal{L}\left(x_{0}, \lambda_{0}\right)(h, h)$ is a Legendre form, and $K_{Y}$ is a polyhedron, then the following condition is necessary and sufficient for quadratic growth,

$$
D_{x^{2}}^{2} \mathcal{L}\left(x_{0}, \lambda_{0}\right)(h, h)>0, \quad \text { for all } h \in C\left(x_{0}\right) \backslash\{0\} .
$$


Proof.Let $x_{0}$ satisfy the quadratic growth condition. Then there exists $\alpha>0$ such that $x_{0}$ is a local solution of the problem

$$
\operatorname{Min}_{x} f(x)-\frac{\alpha}{2}\left\|x-x_{0}\right\|^{2} ; x \in K_{X} ; G(x) \in K_{Y} .
$$

Since $K_{Y}$ is a polyhedron, and therefore $\sigma(\lambda, \mathcal{T}(h))=0,(2.11)$ follows from theorem 2.1.

Conversely, assume that (2.11) holds, while the quadratic growth condition is not satisfied. Then there exists $x_{k} \rightarrow x_{0}$ such that

$$
F\left(x_{k}\right) \leq F\left(x_{0}\right)+o\left(\left\|x_{k}-x_{0}\right\|^{2}\right) .
$$

Set $t_{k}:=\left\|x_{k}-x_{0}\right\|$. Extracting a subsequence if necessary, we may assume that $x_{k}=$ $x_{0}+t_{k} h_{k},\left\|h_{k}\right\|=1$, and $h_{k} \stackrel{w}{\rightarrow} \bar{h}$. Also $\bar{h} \in T_{K_{X}}\left(x_{0}\right)$ since $h_{k} \in \mathcal{R}_{K_{X}}\left(x_{0}\right),(2.12)$ implies that $D F\left(x_{0}\right) \bar{h} \leq 0$, and from $G\left(x_{k}\right) \in K_{Y}$ we deduce that $D G\left(x_{0}\right) \bar{h} \in T_{K_{Y}}\left(G\left(x_{0}\right)\right)$. It follows that $\bar{h}$ is a critical direction.

By the first order optimality condition we have

$$
\left\langle q_{0}, x_{k}-x_{0}\right\rangle \leq 0 \text { and }\left\langle\lambda_{0}, G\left(x_{k}\right)-G\left(x_{0}\right)\right\rangle \leq 0 .
$$

Combining with $D_{x} \mathcal{L}\left(x_{0}, \lambda_{0}\right)+q_{0}=0$, we deduce that

$$
\begin{aligned}
F\left(x_{k}\right)-F\left(x_{0}\right) & \geq \mathcal{L}\left(x_{k}, \lambda_{0}\right)-\mathcal{L}\left(x_{0}, \lambda_{0}\right)+\left\langle q_{0}, x_{k}-x_{0}\right\rangle, \\
& =\frac{t_{k}^{2}}{2} Q_{0}\left(h_{k}\right)+o\left(t_{k}^{2}\right) .
\end{aligned}
$$

Combining with (2.12), it follows that $Q_{0}\left(h_{k}\right) \leq o(1)$. Since $Q_{0}(\cdot)$ is l.s.c., we have $Q_{0}(\bar{h}) \leq$ 0 . Since $\bar{h}$ is critical, this with $(2.11)$ imply that $\bar{h}=0$. It follows that $Q_{0}\left(h_{k}\right) \rightarrow Q_{0}(\bar{h})$. Due to $\left\|h_{k}\right\|=1$ and $\bar{h}=0$, this contradicts the fact that $Q_{0}\left(h_{k}\right)$ is a Legendre form.

\section{Abstract sensitivity analysis}

This section is devoted to the study of the family of perturbed optimization problems

$$
\operatorname{Min}_{x} F(x, u) \text { s.t. } x \in K_{X} ; G(x, u) \in K_{Y} \text {. }
$$

Here $u$ belongs to the Banach space $U, K_{X}$ is a closed convex subset of the Hilbert space $X$, $K_{Y}$ is a polyhedron included in the finite dimensional space $Y$, so that $(C Q A)$ is equivalent to the strict qualification condition (2.6), $f$ and $G$ are twice continuously differentiable mappings from $X \times U$ into $\mathbb{R}$ and $Y$. The Lagrangian of this problem is

$$
\mathcal{L}(x, \lambda, u):=F(x, u)+\langle\lambda, G(x, u)\rangle .
$$

We perform a sensitivity analysis along a path of perturbation variables of the form

$$
u(t):=u_{0}+t u_{1}+\frac{t^{2}}{2} u_{2}+o\left(t^{2}\right)
$$


The following problems may be interpreted as the linearization and the second order expansion of problem $\left(A P_{u}\right)$ at $\left(x_{0}, u_{0}\right)$ along the path $u(t)$, respectively:

$$
\begin{array}{r}
\operatorname{Min}_{h \in X} D f\left(x_{0}, u_{0}\right)\left(h, u_{1}\right) \text { s.t. } h \in T_{K_{X}}\left(x_{0}\right) ; \\
D G\left(x_{0}, u_{0}\right)\left(h, u_{1}\right) \in T_{K_{Y}}\left[G\left(x_{0}, u_{0}\right)\right],
\end{array}
$$

and, $\left(q_{0}, \lambda_{0}\right)$ being the Lagrange multiplier associated with $x_{0}$ :

$$
\operatorname{Min}_{h \in S(L P)} D_{u} \mathcal{L}\left(x_{0}, \lambda_{0}, u_{0}\right) u_{2}+D_{(x, u)^{2}}^{2} \mathcal{L}\left(x_{0}, \lambda_{0}, u_{0}\right)\left(\left(h, u_{1}\right),\left(h, u_{1}\right)\right) ;
$$

Lemma 3.1 Let $x_{0}$ satisfy $(C Q A)$. Then (i) $S(L P)$ is non empty, and

$$
\operatorname{val}(L P)=D_{u} \mathcal{L}\left(x_{0}, \lambda_{0}, u_{0}\right) u_{1},
$$

where $\left(q_{0}, \lambda_{0}\right)$ is the unique Lagrange multiplier associated with $x_{0}$, and

(ii) The set $S(L P) \cap \mathcal{R}_{K_{X}}\left(x_{0}\right)$ is a dense subset of $S(L P)$.

Proof.The dual, in the sense of convex analysis, to the linearized problem $(L P)$, is known to be (e.g. [8])

$$
\operatorname{Max}_{(q, \lambda)} D_{u} \mathcal{L}\left(x_{0}, \lambda, u_{0}\right) u_{1} ; \quad(q, \lambda) \in \Lambda\left(x_{0}\right) .
$$

By lemma 2.4, we know that there exists a unique Lagrange multiplier $\left(q_{0}, \lambda_{0}\right)$, and that the primal and dual values are equal. This proves (3.13). It follows that $h \in X$ is solution of $(L P)$ iff $h \in F(L P)$ and the complementarity conditions

$$
\left\langle q_{0}, h\right\rangle=\left\langle\lambda_{0}, D G\left(x_{0}, u_{0}\right)\left(h, u_{1}\right)\right\rangle=0
$$

are satisfied. In other words, $h \in S(L P)$ iff

$$
h \in T_{K_{X}}\left(x_{0}\right) \cap\left(q_{0}\right)^{\perp} ; \quad D G\left(x_{0}, u_{0}\right)\left(h, u_{1}\right) \in T_{K_{Y}}\left[G\left(x_{0}, u_{0}\right)\right] \cap\left(\lambda_{0}\right)^{\perp} .
$$

By $(C Q A)$ the set of such $h$ is not empty, hence $S(L P)$ is not empty.

(ii) This is a consequence of theorem 2.1(i) applied to problem $(L P)$, once we have checked that problem $(L P)$ itself satisfies the strict semilinearized qualification condition. The expression of the latter (for problem $(L P)$ ) is

$$
0 \in \operatorname{int}\left\{D G(x)\left[\left(T_{K_{X}\left(x_{0}\right)}-h\right) \cap q_{0}^{\perp}\right]-\left(T_{T_{K_{Y}}\left[G\left(x_{0}\right)\right]} D G\left(x_{0}\right) h\right) \cap \lambda_{0}^{\perp}\right\} .
$$

Since $\left(K_{X}-x_{0}\right) \subset T_{K_{X}\left(x_{0}\right)}-h$ and $T_{K_{Y}}\left[G\left(x_{0}\right)\right] \subset T_{T_{K_{Y}}\left[G\left(x_{0}\right)\right]} D G\left(x_{0}\right) h$, this is an obvious consequence of $(C Q A)$.

Theorem 3.1 Assume that

(i) For small enough $t>0$, there exists $x(t)$, o( $\left.t^{2}\right)$-solution of $S\left(A P_{u(t)}\right)$, such that $x(t) \rightarrow x_{0}$.

(ii) The point $x_{0}$ is the unique solution of $\left(A P_{0}\right)$, and satisfies $(C Q A)$ and the second order sufficient optimality condition (2.11), 
(iii) The Hessian $Q_{0}(h):=D_{x^{2}}^{2} \mathcal{L}\left(x_{0}, \lambda_{0}, u_{0}\right)(h, h)$ is a Legendre form over the Hilbert space $X$,

Then

(a) The following expansion for the value function of $\left(A P_{u(t)}\right)$ holds:

$$
\operatorname{val}\left(A P_{u(t)}\right)=\operatorname{val}\left(A P_{u_{0}}\right)+t \operatorname{val}(L P)+\frac{t^{2}}{2} \operatorname{val}(S P)+o\left(t^{2}\right)
$$

(b) One has $x(t)=x_{0}+O(t)$. Any weak limit-point of $t^{-1}\left(x(t)-x_{0}\right)$ is a strong limitpoint, and is solution of $(S P)$. In particular, if $(S P)$ has a unique solution $\bar{h}$, then $x(t)=$ $x_{0}+t \bar{h}+o(t)$.

Proof.Let $Q_{u_{1}}(h):=D_{x^{2}}^{2} \mathcal{L}\left(x_{0}, \lambda_{0}\right)\left(\left(h, u_{1}\right),\left(h, u_{1}\right)\right)$. (Note that this notation is coherent with the definition of $Q_{0}(\cdot)$ given before.) Consider the subproblem

$$
\operatorname{Min}_{h \in S(L P)} D_{u} \mathcal{L}\left(x_{0}, \lambda, u_{0}\right) u_{2}+Q_{u_{1}}(h)-\sigma\left(q_{0}, T_{K_{X}}^{2}\left(x_{0}, h\right)\right) .
$$

Since $K$ is a polyhedron, we have $\sigma\left(\lambda_{0}, T_{K}^{2}\left[G\left(x_{0}, u_{0}\right), D G\left(x_{0}, u_{0}\right)\left(h, u_{1}\right)\right]\right)=0$. It follows from [8, Prop. 2.1] that

$$
\operatorname{val}\left(A P_{u(t)}\right) \leq \operatorname{val}\left(A P_{u_{0}}\right)+t \operatorname{val}(L P)+\frac{t^{2}}{2} \operatorname{val}\left(S P_{\sigma}\right)+o\left(t^{2}\right),
$$

while [8, Prop. 4.3] imply that the right-hand-side of (3.14) is a lower estimate of $\operatorname{val}\left(A P_{u(t)}\right)$. We now prove (3.14) by checking that $\operatorname{val}(S P) \geq \operatorname{val}\left(S P_{\sigma}\right)$. By lemma 3.1, the set $S(L P) \cap$ $\mathcal{R}_{K_{X}}\left(x_{0}\right)$ is a dense subset of $S(L P)$. Also on $S(L P) \cap \mathcal{R}_{K_{X}}\left(x_{0}\right)$ the cost functions of $(S P)$ and $\left(S P_{\sigma}\right)$ coincide. Since $\sigma\left(q_{0}, T_{K_{X}}^{2}\left(x_{0}, h\right)\right) \leq 0$, it follows that

$$
\begin{aligned}
\operatorname{val}(S P) & =\inf _{h \in S(L P) \cap \mathcal{R}_{K_{X}}\left(x_{0}\right)}\left\{D_{u} \mathcal{L}\left(x_{0}, \lambda_{0}, u_{0}\right) u_{2}+Q_{u_{1}}(h)\right\} \\
& =\inf _{h \in S(L P) \cap \mathcal{R}_{K_{X}}\left(x_{0}\right)}\left\{D_{u} \mathcal{L}\left(x_{0}, \lambda_{0}, u_{0}\right) u_{2}+Q_{u_{1}}(h)-\sigma\left(q_{0}, T_{K_{X}}^{2}\left(x_{0}, h\right)\right)\right\} \\
& \geq \operatorname{val}\left(S P_{\sigma}\right),
\end{aligned}
$$

as was to be proved.

(b) By [8, Prop 5.3], we have $x(t)=x_{0}+O(t)$. Let us prove that any weak limit-point of $t^{-1}\left(x(t)-x_{0}\right)$ is a strong limit-point. Let $t_{k} \rightarrow 0^{+}, x_{k}:=x\left(t_{k}\right)$, and $h_{k}:=t_{k}^{-1}\left(x_{k}-x_{0}\right)$ be such that $h_{k} \stackrel{w}{\rightarrow} \bar{h}$. By [8, Prop 5.3], we know that

$$
Q_{u_{1}}\left(h_{k}\right) \rightarrow Q_{u_{1}}(\bar{h}) .
$$

Since $Q_{0}(\cdot)$ is a Legendre form, we have $h_{k} \rightarrow \bar{h}$, as was to be proved. Finally if $(S P)$ has a unique solution $\bar{h}$, it follows that $t^{-1}\left(x(t)-x_{0}\right)$ converges to $\bar{h}$. The conclusion follows. 


\section{Application to state constrained optimal control pro- blems}

\subsection{General results}

In this section we apply the results of the previous sections to some optimal control problems for semilinear elliptic equations. In the sequel of this paper, we denote by $\Omega$ a bounded open subset of $\mathbb{R}^{n}(n \leq 3)$ with Lipschitz boundary $\Gamma$. Given a function $u \in L^{2}(\Omega)$, (we take in this section the standard notations for optimal control problems) we consider the following boundary value problem:

$$
-\Delta y+\phi(x, y)=u \text { in } \Omega, \quad y(x)=0 \text { on } \Gamma,
$$

where $\phi: \bar{\Omega} \times \mathbb{R} \longrightarrow \mathbb{R}$ is a continuous function which is of class $C^{2}$, and such that $\phi_{y}^{\prime}(x, \cdot) \geq 0$, for all $x \in \Omega$.

From now on, the weak solution of (4.15) associated with $u$ will be denoted $y_{u}$. Under the above assumption, we can prove the existence and uniqueness of a solution of (4.15).

Theorem 4.1 For every $u \in L^{2}(\Omega)$, equation (4.15) admits a unique weak solution $y_{u}$ in $H_{0}^{1}(\Omega) \cap C(\bar{\Omega})$, this solution is Hölder continuous and we have

$$
\left\|y_{u}\right\|_{C(\bar{\Omega})} \leq C_{1}\left(1+\|u\|_{L^{2}(\Omega)}\right),
$$

where $C_{1}=C_{1}(\Omega)$ is independent of $u$. Moreover, if we denote by $\mathcal{A}: L^{2}(\Omega) \longrightarrow C(\bar{\Omega})$ the mapping which associates with every control $u$ the weak solution $y_{u}$ of (4.15), then $\mathcal{A}$ is twice continuously Fréchet differentiable, and for every $u, h \in L^{2}(\Omega)$, if we denote $y_{u}=\mathcal{A}(u)$ and $z_{h}=\mathcal{A}^{\prime}(u) h$, then $z_{h}$ is the weak solution of

$$
-\Delta z_{h}+\phi_{y}^{\prime}\left(x, y_{u}\right) z_{h}=h \quad \text { in } \Omega, \quad z_{h}=0 \quad \text { on } \Gamma .
$$

Proof.The above theorem is a collection of known results for semilinear elliptic equations (see $[7,6,15]$ and the general references $[1,2,5])$.

Consider the following control constraints:

$$
L_{+}^{2}(\Omega):=\left\{u \in L^{2}(\Omega) \mid u(x) \geq 0 \text { a.e. on } x \in \Omega\right\} .
$$

Let us also consider a family of functions $G_{j}$ of class $C^{2}: L^{2}(\Omega) \rightarrow \mathbb{R}$, for $1 \leq j \leq m$. We consider the following optimal control problem:

$$
\operatorname{Min}\left\{J\left(y_{u}, u\right) \mid u \in L_{+}^{2}(\Omega), G_{j}(u) \leq 0 \text { for } 1 \leq j \leq m\right\},
$$

where

$$
F(u):=\frac{1}{2} \int_{\Omega}\left(y_{u}(x)-y_{d}(x)\right)^{2} d x+\frac{N}{2} \int_{\Omega} u(x)^{2} d x,
$$


with $y_{d}$ is a given function in $L^{2}(\Omega)$, and $N>0$. The adjoint state $p_{u}^{0}$ associated with $u$ is defined as the unique solution in $H^{2}(\Omega)$ of the system

$$
-\Delta p_{u}^{0}+\phi_{y}^{\prime}\left(x, y_{u}\right) p_{u}^{0}=y_{u}-y_{d} \text { in } \Omega, \quad p_{u}^{0}=0 \text { on } \Gamma .
$$

It is known that $u \rightarrow F(u)$ is a $C^{2}$ mapping with derivative

$$
D F(u)=N u+p_{u}^{0} .
$$

We will detail later the cases when $G_{j}(u)$ are some punctual or integral functions of the state.

Let $\bar{u}$ be an optimal solution of problem $(\mathcal{P})$. Set

$$
\begin{aligned}
J_{+} & =\left\{j \in\{1, \cdots, m\} \mid G_{j}(\bar{u})<0\right\}, \\
J_{0} & =\left\{j \in\{1, \cdots, m\} \mid G_{j}(\bar{u})=0\right\}, \\
J_{-} & =\left\{j \in\{1, \cdots, m\} \mid G_{j}(\bar{u})=0, \bar{\lambda}_{j}>0\right\} .
\end{aligned}
$$

Then $\left(J_{0}, J_{-}, J_{+}\right)$is a partition of $\{1, \cdots, m\}$. Problem $(\mathcal{P})$ can be written as follows

$$
\operatorname{Min}\left\{F(u) \mid u \in L_{+}^{2}(\Omega), G(u) \in \mathbb{R}_{-}^{m}\right\} .
$$

In addition (see e.g. [10,3]) Robinson's constraint qualification assumption is equivalent to

$$
\exists v \in L_{+}^{2}(\Omega), \quad G_{j}(\bar{u})+D G_{j}(\bar{u})(v-\bar{u})<0 .
$$

Therefore we obtain the following (classical) expression of the first order optimality system.

Theorem 4.2 Assume that $\bar{u}$ is a local solution of $(P)$ satisfying (4.18). Denote by $\bar{y}$ and $\bar{p}$ the state and adjoint state associated with $\bar{u}$. Then there exist Lagrange multipliers $(\bar{q}, \bar{\lambda}) \in L^{2}(\Omega) \times \mathbb{R}^{m}$ such that:

$$
\begin{aligned}
& \bar{\lambda}_{j} \geq 0,1 \leq j \leq m, \text { and } \bar{\lambda}_{j}=0 \text { if } G_{j}(\bar{u})<0, \\
& N \bar{u}+\bar{p}+\sum_{j=1}^{m} \bar{\lambda}_{j} D G_{j}(\bar{u})+\bar{q}=0 ; \quad\langle\bar{q}, u-\bar{u}\rangle \leq 0, \quad \forall u \in L_{+}^{2}(\Omega) .
\end{aligned}
$$

Since $L_{+}^{2}(\Omega)$ is polyhedric in $L^{2}(\Omega)$ (see e.g. $\left.[18,25]\right),(\mathcal{P})$ is of the form $(A P)$, with

$$
X=L^{2}(\Omega), Y=\mathbb{R}^{m}, K_{X}=L_{+}^{2}(\Omega), K_{Y}=\mathbb{R}_{-}^{m} .
$$

We now discuss the strict semilinearized qualification condition $(C Q A)$. We need a notation for the contact set of $\bar{u}$ and its complement (defined up to a null measure set):

$$
\begin{gathered}
\Omega_{-}(\bar{u}):=\{x \in \Omega ; \bar{q}(x)>0\}, \quad \Omega_{0}(\bar{u}):=\{x \in \Omega ; \bar{u}(x)=0\}, \\
\Omega_{+}(\bar{u}):=\{x \in \Omega ; \bar{u}(x)>0\} .
\end{gathered}
$$


Since $\Omega_{-}(\bar{u}) \subset \Omega_{0}(\bar{u})$, we have:

$$
T_{L_{+}^{2}(\Omega)}(\bar{u}) \cap \bar{q}^{\perp}=\left\{h \in L^{2}(\Omega) ; h \geq 0 \text { on } \Omega_{0} ; h=0 \text { on } \Omega_{-}(\bar{u})\right\} .
$$

Therefore, the strict qualification condition is identical to the qualification condition when changing inequalities $G_{j}(\cdot) \leq 0$ into equalities, for $j \in J_{-}$, and adding the constraint that the control is zero on $\Omega_{-}(\bar{u})$.

Lemma 4.1 Let $\bar{u} \in F(\mathcal{P})$, with associated Lagrange multiplier $(\bar{q}, \bar{\lambda})$. Then the three conditions below are equivalent:

(i) The strict qualification condition $(C Q A)$ is satisfied.

(ii) The following conditions hold:

$$
\left\{\begin{array}{l}
\text { (i) }\left\{D G_{i}(\bar{u}) h ; i \in J_{-} ; h \in\left(T_{L_{+}^{2}(\Omega)}(\bar{u}) \cap \bar{q}^{\perp}\right)\right\} \text { is onto, } \\
\text { (ii) } \exists h \in\left(\mathcal{R}_{L_{+}^{2}(\Omega)}(\bar{u}) \cap \bar{q}^{\perp}\right) ; D G_{i}(\bar{u}) h=0, i \in J_{-} ; D G_{i}(\bar{u}) h<0, i \in J_{0} \backslash J_{-} .
\end{array}\right.
$$

(iii) There exists no $(\tilde{q}, \tilde{\lambda}) \in L^{2}(\Omega) \times \mathbb{R}^{m}$, with $\tilde{\lambda} \neq 0$, satisfying the following relations:

$$
\left\{\begin{array}{l}
\text { (i) } \tilde{\lambda}_{i}=0, i \in J_{+} ; \tilde{\lambda}_{i} \geq 0, i \in J_{0} \backslash J_{-}, \\
\text {(ii) } \tilde{q}(x)=0 \text { on } \Omega_{+}(\bar{u}), \quad \tilde{q}(x) \leq 0 \quad \text { on } \Omega_{0}(\bar{u}) \backslash \Omega_{-}(\bar{u}) ; \\
\text { (iii) } \tilde{q}+\sum_{1 \leq i \leq m} \tilde{\lambda}_{i} D G_{i}(\bar{u})=0 .
\end{array}\right.
$$

Proof.By the definition, $(C Q A)$ holds iff, for any $z \in \mathbb{R}^{m}$, close enough to 0 , there exists $h \in\left(L_{+}^{2}(\Omega)-\bar{u}\right) \cap \bar{q}^{\perp}$ satisfying the following relations:

$$
\text { (i) } D G_{i}(\bar{u}) h=z_{i}, i \in J_{-} ; \quad \text { (ii) } D G_{i}(\bar{u}) h \leq z_{i}, i \in J_{0} \backslash J_{-} \text {. }
$$

It follows from (4.23(i)) that the set

$$
\left\{D G_{i}(\bar{u}) h ; \quad i \in J_{-} ; \quad h \in\left(\mathcal{R}_{L_{+}^{2}(\Omega)}(\bar{u}) \cap \bar{q}^{\perp}\right)\right\} \text { is onto. }
$$

This implies that $(4.21(\mathrm{i}))$ is a necessary condition for $(C Q A)$. Then taking $z_{i}=0, i \in J_{-}$, and $z_{i}<0, i \in J_{0} \backslash J_{-}$, we deduce that (4.21) is a necessary condition for $(C Q A)$, i.e. $(C Q A) \Rightarrow(4.21)$. We end the proof by showing that $(4.21) \Rightarrow(4.22) \Rightarrow(C Q A)$.

Assume that $(C Q A)$ does not hold. Then the convex cone

$$
E:=\left\{D G(\bar{u}) h-z ; h \in \mathcal{R}_{L_{+}^{2}(\Omega)}(\bar{u}) \cap \bar{q}^{\perp} ; z_{i} \leq 0, i \in J_{0}, z_{i}=0, i \in J_{-}\right\}
$$

is not equal to $\mathbb{R}^{m}$. Since the latter is a finite dimensional space, the closure of $E$ is not equal to $\mathbb{R}^{m}$. By the Hahn-Banach theorem, since $E$ is a cone, there exists $\tilde{\lambda} \in \mathbb{R}^{m}, \tilde{\lambda} \neq 0$, such that $\langle\tilde{\lambda}, y\rangle \geq 0$, for all $y \in E$. It follows that (4.22(i)) holds, while $\tilde{q}$ defined by (4.22(iii)) is such that

$$
-\langle\tilde{\lambda}, D G(\bar{u}) h\rangle=\int_{\Omega} \tilde{q}(x) h(x) d x \leq 0, \quad \forall h \in\left(L_{+}^{2}(\Omega)-\bar{u}\right) \cap \bar{q}^{\perp} .
$$


Since the polar of the intersection of two closed convex cones is the closure of the sum of their polar cones, we have that

$$
\tilde{q} \in\left(\left(L_{+}^{2}(\Omega)-\bar{u}\right) \cap \bar{q}^{\perp}\right)^{-}=\overline{\left(L_{+}^{2}(\Omega)-\bar{u}\right)^{-}+\mathbb{R} \bar{q}} .
$$

Relation (4.22(ii)) follows.

Finally, suppose that (4.21) holds, but (4.22) does not hold. Let $(\tilde{q}, \tilde{\lambda})$ satisfy (4.22). Then (4.24) holds. It follows that for each $h \in \mathcal{R}_{L_{+}^{2}(\Omega)}(\bar{u}) \cap \bar{q}^{\perp}$

$$
0 \leq-\int_{\Omega} \tilde{q}(x) h(x) d x=\sum_{1 \leq i \leq m} \tilde{\lambda}_{i} D G_{i}(\bar{u}) h .
$$

This and (4.21(ii)) imply $\tilde{\lambda}_{i}=0$, for all $i \in J_{0} \backslash J_{-}$. Then, since $L_{+}^{2}(\Omega)$ is polyhedric, with (4.21(i)) we obtain $\tilde{\lambda}_{i}=0$, for all $i \in J_{-}$, in contradiction with the fact that $\tilde{\lambda} \neq 0$.

Denote by $L^{2}\left(\Omega_{+}(\bar{u})\right)$ the Hilbert space of functions of $L^{2}(\Omega)$ that are a.e. null outside $\Omega_{+}(\bar{u})$. Fom the above lemma, we deduce the following corollary, similar to [15, Thm 5.2].

Corollary 4.1 A sufficient condition for $(C Q A)$ is that the restriction of $D G(\bar{u})$ to $L^{2}\left(\Omega_{+}(\bar{u})\right)$, with image space $\mathbb{R}^{m}$, is onto.

We now discuss second order optimality conditions. Since $Q_{0}(\cdot)$ is a Legendre form, we have the following result, that is an immediate consequence of Theorem 2.2. Note that the assumption, that the Hessians $D^{2} G(\bar{u})$ are weakly continuous, is typically satisfied if $G$ represents state constraints, as will be the case in the examples to be seen later. The expression of the Lagrangian for problem $(\mathcal{P})$ is

$$
\mathcal{L}(u, \lambda):=F(u)+\sum_{i=1}^{m} \lambda_{i} G_{i}(u) .
$$

Theorem 4.3 Let $\bar{u}$ be an optimal solution of $(\mathcal{P})$, with associated Lagrange multiplier $(\bar{q}, \bar{\lambda})$, satisfy condition $(C Q A)$. Assume that the Hessians $D^{2} G(\bar{u})$ are weakly continuous. Then $\bar{u}$ satisfies the quadratic growth condition iff

$$
D_{u u}^{2} \mathcal{L}(\bar{u}, \bar{\lambda})(h, h)>0, \quad \forall h \in C(\bar{u}), \quad h \neq 0 .
$$

\subsection{Problems with finitely many punctual state constraints}

We consider in this subsection the case when the functions $G_{j}, 1 \leq j \leq m$, are defined by

$$
G_{j}(u)=y_{u}\left(x_{j}\right)-b_{j} .
$$

Here $b \in \mathbb{R}^{m}$ and $x_{j} \in \Omega, 1 \leq j \leq m$, are given. We denote $\bar{y}:=y_{\bar{u}}$. A simple consequence of lemma 4.1 follows: 
Lemma 4.2 Assume that $\Omega_{+}(\bar{u})$ has a non empty interior. Then the strict semilinearized qualification condition $(C Q A)$ is satisfied.

Proof.If the conclusion does not hold, then by lemma 4.1, there exists $(\tilde{q}, \tilde{\lambda}) \in L^{2}(\Omega) \times$ $\mathbb{R}^{m}$, with $\tilde{\lambda} \neq 0$, satisfying (4.22). It is a classical result (see e.g. [12]) that $\tilde{q} \in L^{2}(\Omega) \cap$ $W^{1, s}(\Omega)$, for all $s<n /(n-1)$, and is the unique solution in $W^{1,1}(\Omega)$ of

$$
-\Delta \tilde{q}+\phi_{y}^{\prime}(x, \bar{y}) \tilde{q}=-\sum_{1 \leq i \leq m} \lambda_{i} \delta\left(x_{i}\right) \text { in } \Omega, \quad \tilde{q}=0 \text { on } \Gamma .
$$

Here $\delta\left(x_{i}\right)$ stands for the Dirac measure at point $x_{i}$. Since $\tilde{q}=0$ on the interior of $\Omega_{+}$, and the latter is non empty, we have by the unique extension theorem [29] that $\tilde{q}=0$ over $\Omega$ except perhaps at the points $x_{j}$. But this implies $\tilde{\lambda}=0$, in contradiction with the hypothesis.

We now state the characterization of quadratic growth. By $z_{h}$ we denote the solution of the linearized equation (4.16) with $y_{u}=\bar{y}$ and r.h.s. $h$. As a consequence of theorem 4.3, we have:

Theorem 4.4 Let $\bar{u}$ be a feasible point of $(\mathcal{P})$, with associated Lagrange multiplier $(\bar{q}, \bar{\lambda})$, and assume that the interior of $\Omega_{+}$is non empty. Then $\bar{u}$ satisfies the quadratic growth condition iff there exists $\bar{p} \in W^{1, s}(\Omega)$, for all $s<n /(n-1)$, such that

$$
\begin{aligned}
& \bar{\lambda}_{j} \geq 0,1 \leq j \leq m, \text { and } \bar{\lambda}_{j}=0 \text { if } G_{j}(\bar{u})<0, \\
& -\Delta \bar{p}+\phi_{y}^{\prime}(x, \bar{y}) \bar{p}=\bar{y}-y_{d}+\sum_{j=1}^{m} \bar{\lambda}_{j} \delta\left(x_{j}\right) \quad \text { in } \Omega, \quad \bar{p}=0 \quad \text { on } \Gamma ; \\
& \int_{\Omega}(N \bar{u}(x)+\bar{p}(x))(u(x)-\bar{u}(x)) d x \geq 0, \quad \forall u \in L_{+}^{2}(\Omega),
\end{aligned}
$$

and such that, for all $h \in C(\bar{u}), h \neq 0$, and $z_{h}$ solution of (4.16) (in which $\bar{y}=y_{\bar{u}}$ ):

$$
\int_{\Omega}\left(N h(x)^{2}+z_{h}^{2}(x)^{2}-\bar{p}(x) \phi_{y}^{\prime \prime}(x, \bar{y}) z_{h}(x)^{2}\right) d x>0 .
$$

We now discuss sensitivity of the solution of the optimal control problem with respect to the target $y_{d}$. Therefore we denote

$$
F\left(u, y_{d}\right):=\frac{1}{2} \int_{\Omega}\left(y_{u}(x)-y_{d}(x)\right)^{2} d x+\frac{N}{2} \int_{\Omega} u(x)^{2} d x .
$$

Consider a target path, where $t \geq 0$,

$$
y_{d}(t)=y_{d 0}+t y_{d 1}+\frac{t^{2}}{2} y_{d 2}+o\left(t^{2}\right)
$$

Note that

$$
D_{y_{d}} F\left(u, y_{d 0}\right) y_{d 1}=-\int_{\Omega}\left(\bar{y}-y_{d 0}\right)(x) y_{d 1}(x) d x \text {. }
$$


The subproblems to be considered here, corresponding to $(L P)$ and $(S P)$, are

$$
\begin{gathered}
\operatorname{Min}_{h \in L^{2}(\Omega)} \int_{\Omega}(N \bar{u}+\bar{p})(x) h(x) d x-\int_{\Omega}\left(\bar{y}-y_{d 0}\right)(x) y_{d 1}(x) d x \\
\text { s.t. } h \geq 0 \text { on } \Omega_{0}(\bar{u}) ; z_{h}\left(x_{i}\right) \leq 0, i \in J_{0},
\end{gathered}
$$

and, denoting by $z_{h}$ the solution of (4.16) (in which $y_{u}=\bar{y}$ ):

$$
\operatorname{Min}_{h \in S(\overline{L P})} D^{2} F\left(\bar{u}, y_{d 0}\right)\left(\left(h, y_{d 1}\right),\left(h, y_{d 1}\right)\right)-\int_{\Omega}\left(\bar{y}-y_{d 0}\right)(x) y_{d 2}(x) d x .
$$

(An expression of the Hessian of $F$ in term of $\bar{p}$ and $z_{h}$ is given in [6].)

Theorem 4.5 Assume that $\bar{u}$ is the unique solution of $(A P)$, and satisfies $(C Q A)$ as well as the second order sufficient optimality condition (4.29). Then

(a) The following expansion for the value function of $\left(A P_{u(t)}\right)$ holds:

$$
\operatorname{val}\left(A P_{u(t)}\right)=\operatorname{val}\left(A P_{\bar{u}}\right)+t \operatorname{val}(\overline{L P})+\frac{t^{2}}{2} \operatorname{val}(\overline{S P})+o\left(t^{2}\right),
$$

(b) Let $u(t)$ be a path of $o\left(t^{2}\right)$-solutions. Then one has $u(t)=\bar{u}+O(t)$. Any weak limit-point of $t^{-1}(u(t)-\bar{u})$ is a strong limit-point, and is solution of $(\overline{S P})$. In particular, if $(\overline{S P})$ has a unique solution $\bar{h}$, then $u(t)=\bar{u}+t \bar{h}+o(t)$.

Proof.It is easy to check that the solutions of the perturbed problem are uniformly bounded, and that they strongly converge in $L^{2}(\Omega)$ to $\bar{u}$, see e.g. [6]. In addition the Hessian of the Lagrangian, that is equal to the Hessian of the cost, is a Legendre form. Therefore the conclusion is a consequence of theorem 3.1.

\subsection{Problems with integral state constraints}

We consider in this subsection the case when the functions $G_{j}(u) 1 \leq j \leq m$, are defined by

$$
G_{j}(u)=\int_{\Omega} g_{j}\left(y_{u}(x), x\right) d x .
$$

The functions $g_{j}(u)$ are assumed to be twice continuously differentiable functions $\mathbb{R} \times \bar{\Omega} \rightarrow$ $\mathbb{R}$. Then $G(\cdot)$ is itself a $C^{2}$ mapping. We know that the derivative of $u \rightarrow G_{j}(u)$, viewed as a function $L^{2}(\Omega) \rightarrow \mathbb{R}$, is $p_{j}(u) \in H^{2}(\Omega)$ solution of

$$
-\Delta p_{j}+\phi_{y}^{\prime}\left(x, y_{u}\right) p_{j}=D_{y} g_{j}\left(y_{u}(x), x\right) \text { in } \Omega, \quad p_{j}=0 \text { on } \Gamma \text {. }
$$

A simple consequence of lemma 4.1 follows: 
Lemma 4.3 The strict qualification condition $(C Q A)$ is satisfied iff the following system has no solution $(\tilde{q}, \tilde{\lambda}) \in W^{1, s}(\Omega) \times \mathbb{R}^{m}$ :

$$
\begin{aligned}
& -\Delta \tilde{q}+\phi_{y}^{\prime}(x, \bar{y}) \tilde{q}=-\sum_{1 \leq i \leq m} \lambda_{i} D_{y} g_{j}(\bar{y}(x), x) \quad \text { in } \Omega, \quad \tilde{q}=0 \quad \text { on } \Gamma . \\
& \tilde{q}(x)=0, \quad \text { on } \Omega_{+}(\bar{u}), \quad \tilde{q}(x) \geq 0 \quad \text { on } \Omega_{0}(\bar{u}) \backslash \Omega_{-}(\bar{u}), \\
& \lambda \neq 0 ; \quad \lambda_{i}=0, i \in J_{+}, \quad \lambda_{i} \geq 0, i \in J_{0} \backslash J_{-} .
\end{aligned}
$$

Let us give an example of such integral constraints for which condition $(C Q A)$ can be checked. Let $b \in \mathbb{R}^{m}$, and $a_{j} \in C(\bar{\Omega})$, for $1 \leq j \leq m$, with $a_{j}(x)$ of constant sign over its support $\Omega_{j}:=\operatorname{supp}\left(a_{j}\right)$. Assume that these supports satisfy the following geometric relation:

$$
\Omega_{i} \cap \Omega_{j}=\emptyset \text { for } i \neq j ; \quad \Omega \backslash\left(\cup_{1 \leq j \leq m} \Omega_{j}\right) \text { is connected. }
$$

We consider the case when

$$
g_{j}(u):=\int_{\Omega} a_{j}(x) y_{u}(x) d x-b_{j} .
$$

We assume also the following:

There exists $\Omega_{*}$, open subset of $\Omega_{+}(u)$, such that $\Omega_{*} \cap \Omega_{j}=\emptyset, 1 \leq j \leq m$.

Lemma 4.4 Under the above hypotheses, the strict qualification condition (CQA) is satisfied.

Proof.If the conclusion does not hold, then there exists $(\tilde{q}, \tilde{\lambda})$ satisfying the condition of lemma 4.3 , and in particular

$$
-\Delta \tilde{q}+\phi_{y}^{\prime}(x, \bar{y}) \tilde{q}=-\sum_{j \in J_{0} \cup J_{-}} \tilde{\lambda}_{j} a_{j} \text { in } \Omega, \quad \tilde{q}=0 \quad \text { on } \Gamma,
$$

as well as $\tilde{q}=0$ on $\Omega_{*}$. Set $A:=\Omega \backslash\left(\cup_{1 \leq j \leq m} \Omega_{j}\right)$. Then $A$ is a connected open set that contains $\Omega_{*}$. Since $\Omega_{*}$ is open, and $\tilde{q}=0$ on $A$, by the unique extension theorem [29] we obtain $\tilde{q}=0$ on $A$, hence on $\partial \Omega_{j}$, for all $1 \leq j \leq m$. Let $j$ be such that $\tilde{\lambda}_{j} \neq 0$. Let $A_{j}$ be the interior of $\Omega_{j}, 1 \leq j \leq m$, and let

$$
B_{j}:=\left\{x \in \Omega ; \operatorname{dist}\left(x, A_{j}\right) \leq \varepsilon\right\} .
$$

Take $\varepsilon>0$ so small that $B_{j} \backslash A_{j}$ does not intersect $\Omega_{i}$, for $i \neq j$. Then $\tilde{q}$ satisfies

$$
-\Delta \tilde{q}+\phi_{y}^{\prime}\left(x, y_{u}\right) \tilde{q}=-\tilde{\lambda}_{j} a_{j} \text { in } B_{j}, \quad \tilde{q}=0 \text { on } \partial B_{j} .
$$

This equation has a unique solution in $H_{0}^{1}\left(B_{j}\right)$. Since $a_{j}$ is of constant sign, $\tilde{q}$ is nonzero over the interior of $B_{j}$. But this is impossible, since the latter contains a nonempty open set included in $A$.

Whenever $(C Q A)$ holds, we can state a characterization of quadratic growth. We omit the statement since it is similar to theorem 4.2. 


\section{Conclusion and possible extensions}

Our theoretical results extend those in [6], that discuss problems with polyhedric control constraints only. We were able to give an application of these results for control and state constrained optimal control problems, when the number of state constraints is finite.

For technical reasons we discussed only the case when the space dimension $n$ is less or equal 3. Extension of these results in the case $n>3$ seems possible by combining the technique of this paper with the two norms approach $[6,22]$. The latter would also allow to extend our results to the case of boundary control, or to problems with a parabolic state equation.

It seems also possible to extend our results to the case when $K_{Y}$ is not a polyhedron, taking advantage of the results in [9]. For instance, the set of semi definite positive matrices is a closed convex set that satisfies hypothesis (ii) of theorem 2.1. On the other hand, the case of a punctual state constraint at every point of the domain $\Omega$ seems out of reach, since the strict qualification condition is probably not satisfied in that case.

\section{References}

[1] R.A. Adams, Sobolev spaces. Academic Press, New York, 1975.

[2] S. Agmon, A. Douglis, L. Nirenberg, Estimates near the boundary for solutions of elliptic partial differential equations satisfying general boundary conditions I

Communications on Pure and Applied Mathematics 12 (1959), pp. 623-727

[3] J.J. Alibert, J.P. Raymond, A Lagrange multiplier theorem for control problems with state constraints, to appear.

[4] L. BARBet, Analyse de sensibilité différentielle des solutions optimales d'inéquations variationnelles en dimension infinie, Comptes Rendus Acad. Sci. Paris, t. 315 (1992), Série I, pp. 1179-1182.

[5] H. BrÉzis, Problèmes unilatéraux. J. Mathématiques pures et appliquées 51 (1972), pp. $1-168$.

[6] J. F. Bonnans, Second order analysis for control constrained optimal control problems of semilinear elliptic systems. J. Applied Mathematics and Optimization, to appear.

[7] J. F. Bonnans and E. Casas, An extension of Pontryagin's principle for stateconstrained optimal control of semilinear elliptic equations and variational inequalities. SIAM J. Control Optimization 33 (1995), pp. 274-298.

[8] J.F. Bonnans And R. Cominetti, Perturbed optimization in Banach spaces I: a general theory based on a weak directional constraint qualification. SIAM J. Control Optimization 34(1996), pp. 1151-1171. 
[9] J.F. Bonnans and R. Cominetti And A. Shapiro, Second order optimality conditions based on parabolic second order tangent sets. SIAM J. Optimization, to appear.

[10] J.F. Bonnans And A. Shapiro, Optimization problems with perturbations: A guided tour. SIAM Review, to appear.

[11] J.F. Bonnans And A. Shapiro, Perturbation analysis of optimization problems. Book to appear.

[12] E. CASAS, Control of an elliptic problem with pointwise state constraints. SIAM J. Control Optimization 24(1986), 1309-1318.

[13] E. CaSAs, J.P. RAYMOnd, H. Zidani, Optimal control problem governed by semilinear elliptic equations with integral control constraints and pointwise state constraints, in International Conference on Control and Estimations of Distributed Parameter Systems, ISNM, W. Desch and F. Kapple Eds., Birkhaüser-Verlag, 1997.

[14] E. Casas, F. Tröltzsch and A. Unger, Second order sufficient optimality conditions for a nonlinear elliptic boundary control problem, Zeitschrift für Analysis und ihre Anwendungen 15 (1996), pp. 687-707.

[15] E. Casas And F. TRÖltzsch, Second order necessary optimality conditions for some state-constrained control problems of semilinear elliptic equations, Preprint 96-9 (1996), Fakultät für Mathematik, Technische Universität Chemnitz-Zwickau, D-09107 Chemnitz, Germany.

[16] R. Cominetti, Metric regularity, tangent sets and second order optimality conditions, Applied Mathematics and Optimization, 21 (1990), pp. 265-287.

[17] D. Gilbarg and N.S. Trudinger, Elliptic partial differential equations of second order. Springer Verlag, Berlin, 1983.

[18] A. Haraux, How to differentiate the projection on a convex set in Hilbert space. Some applications to variational inequalities, J. Mathematical Society of Japan (29) (1977), pp. $615-631$.

[19] A. D. Ioffe And V. M. Tinomirov, Theory of Extremal Problems, North-Holland Publishing Company, Amsterdam, 1979.

[20] J. L. Lions, Contrôle optimal de systèmes gouvernés par des équations aux dérivées partielles. Dunod, Paris, 1968.

[21] K. Malanowski, Second order conditions and constraint qualifications in stability and sensitivity analysis of solutions to optimization problems in Hilbert spaces, Applied Mathematics and Optimization, 25 (1992), pp. 51-79. 
[22] K. MALANOWSKI, Two-norm approach in stability and sensitivity analysis of optimization and optimal control problems, Advances in Mathematical Sciences and Applications, 2 (1993), pp. 397-443.

[23] K. Malanowski, Stability and sensitivity of solutions to nonlinear optimal control problems. Applied Mathematics and Optimization, 32 (1995), pp. 111-141.

[24] H. Maurer AND J. Zowe, First and second-order necessary and sufficient optimality conditions for infinite-dimensional programming problems, Mathematical Programming, 16 (1979), pp. 98-110.

[25] F. Mignot, Contrôle dans les inéquations variationelles elliptiques, J. Functional Analysis 22 (1976), 25-39.

[26] S.M. RoBinson, Strongly regular generalized equations, Mathematics of Operations Research 5 (1980), pp. 43-62.

[27] R.T. Rockafellar, Conjugate Duality and Optimization, Regional Conference Series in Applied Mathematics, SIAM, Philadelphia, 1974.

[28] A. Shapiro, Perturbation analysis of optimization problems in Banach spaces, Numerical Functional Analysis and Optimization, 13 (1992), pp. 97-116.

[29] J.C. Saut and B. Scheurer, Sur l'unicité du problème de Cauchy et le prolongement unique pour des équations elliptiques à coefficients non localement bornés, J. Differential Equations, 43(1982), 28-43.

[30] J. Sokolowski, Sensitivity analysis of control constrained optimal control problems for distributed parameter systems. SIAM J. Control Optimization 25 (1987), pp. 1542-1556.

[31] J. Zowe AND S. KuRCYUSZ, Regularity and stability for the mathematical programming problem in Banach spaces, Applied Mathematics and Optimization, 5 (1979), pp. 49-62. 
Unit`e de recherche INRIA Lorraine, Technopôle de Nancy-Brabois, Campus scientifique, 615 rue du Jardin Botanique, BP 101, 54600 VILLERS LÈS NANCY

Unit'e de recherche INRIA Rennes, Irisa, Campus universitaire de Beaulieu, 35042 RENNES Cedex Unit'e de recherche INRIA Rhône-Alpes, 655, avenue de l'Europe, 38330 MONTBONNOT ST MARTIN Unit'e de recherche INRIA Rocquencourt, Domaine de Voluceau, Rocquencourt, BP 105, 78153 LE CHESNAY Cedex Unit ${ }^{\prime} e$ de recherche INRIA Sophia-Antipolis, 2004 route des Lucioles, BP 93, 06902 SOPHIA-ANTIPOLIS Cedex

Éditeur

INRIA, Domaine de Voluceau, Rocquencourt, BP 105, 78153 LE CHESNAY Cedex (France) http://www.inria.fr ISSN 0249-6399 